\title{
Use of Interactive Games in Healthcare
}

Hassan A. Aziz*

College of Arts and Sciences, Qatar University, Doha, Qatar

\begin{abstract}
Interactive health games (IHG) are fun, experimental, challenging as well as powerful tools that have the potential to change the patients' behaviour, attitudes and improve their health. IHG involve well-designed intent challenges and follow roles moving toward a goal. In the last decade, a wide variety of IHG has been developed. The classification of these games is based on game subject, health subject and player subject. Along with the potential future utilization of IHG, there are limitations to a wider application such as the disparity in the player's cognitive abilities and the need to identify the kinds of learning and training support.
\end{abstract}

\section{Keywords: Cognitive; Health; Behaviour}

\section{Introduction}

Electronic games have expanded beyond the boundaries of entertainment to change many peoples' lives in a fundamental way. They are now introduced as powerful tools for learning and healthcare delivery. Interactive health games (IHG) are attractive, enjoyable, experimental and challenging, and can change the player's attitudes and behaviours that could subsequently improve their health. Players of well-designed IHG exhibited considerable improvement in their skills, behaviours, and health related knowledge [1]. An interactive game is defined as an experience that involves following rules through an assigned challenge, moving toward a goal, and a well-defined end [2]. Users choose to enter an environment created by games in order to overcome problems or to achieve a goal within the game. In fact, the interactive nature of the games is emphasized to maintain continuous recurrent game cycles.

\section{Serious interactive games}

Serious interactive games can be used to impact factors such as disease management and health education. Additionally, studies have shown that using interactive computer games positively affect elderly adults, specifically by improving levels of psychological and mental health. All games include [3] three essential elements. The first element is the rule of the game. Game rules create the pattern and connect the player with game. Games may have different levels of difficulties or obstructions known as challenges. Good actions are rewarded in order to encourage and motivate the player to reach the game goal and spend more time playing. The second element is the interaction of the player with the game. Interaction is the player's action to start some activity, which can be physical (e.g. mouse, typing or touchpad), visual, or dialogue exchange. Specific tools give input to the gaming system and support this interaction represented by accessories or equipment connected to the game. Finally, the game must have an objective that is intended to be accomplished or reached. Objectives can be explicit or implicit. Explicit objective is the mere entertainment of the game. On the other hand, games with an implicit objective aim at gaining knowledge, increasing abilities and skills, and obtaining experience. Games with an implicit objective are known as serious interactive games [4].

\section{Serious interactive games core components}

The development of serious interactive games involves different processes and technologies. The developing team may consist of graphic designers, managers, programmers, content providers and researchers, amongst others. The team has to determine the technologies, tools and the contents that have to be used in the game. The second core component is identifying the game genre and objectives. Third, the specification of the target player which can be designated by contexts such as playing skill (casual, newbie and hard core), status of player (elderly and children) and playing style (achiever, socializer and explorer). Many [4] serious health games in the last decade have been developed. Those games deal with a wide variety of aspects such as cardiopulmonary resuscitation (CPR), surgeon training and patient care. The number of serious health games is increasing dramatically since their introduction in 2004 reaching over 55 games in 2012 [4].

\section{IHG educational tool}

Researchers found that the game-based approach is much better than educational videotape to raise users' self-management efficacy. Interactive games stimulate active involvement of patients with different conditions, making the learning process more enjoyable with boundless practice chances and where players can engage in behavioral rehearsal [2]. Moreover, a feasibility study showed that in older adults interactive video games increase their cognitive, physical and social activity without the need for skilled specialists. Novel [5], interactive and fun games are used in the education of health care provider to promote learning without facing incorrect decisions consequences made in clinical setting and to reduce anxiety from stressful clinical situations. The games can teach complex subjects without using expensive clinical equipment or exposing the patient to risk. They actively involve large groups in making choices and participating in peer teaching, thus promoting teamwork and team building, challenging and sharing knowledge and experiences with each other. The games can ultimately identify gaps, aid in revisions and strengthen the links between practice and theory. IHG [6] have been created by a variety of organizations, both governmental and non-profit. The intended audiences include health care professionals and consumers, including children. For example, the Nobel Foundation provides games [7] to test knowledge of the immune system, electrocardiograms, or blood types. There are several food safety games for children such as the American Academy of Allergy, Asthma and Immunology's stinging insect matching game.

*Corresponding author: Hassan A. Aziz, PhD, MLS(ASCP)cm, FACSs, College of Arts and Sciences, Qatar University, P.O. Box 2713, Doha, Qatar, Tel: +974-44034783; E-mail: Hassan.Aziz@qu.edu.qa

Received February 19, 2018; Accepted February 24, 2018; Published February 26, 2018

Citation: Aziz HA (2018) Use of Interactive Games in Healthcare. J Health Med Informat 9: 304. doi: 10.4172/2157-7420.1000304

Copyright: @ 2018 Aziz HA. This is an open-access article distributed under the terms of the Creative Commons Attribution License, which permits unrestricted use, distribution, and reproduction in any medium, provided the original author and source are credited. 


\section{Limitations}

Despite the potential future utilization for IHG in healthcare and education settings, there are limitations regarding their use. Creating the suitable material and learning process requires a lot of time and there is a high risk for cognitive load for the patient due to the amount of information that has to be processed simultaneously. The gaming nature requires element's such as fun elements or interesting storyline that might be destructive while "seductive details" may not be suitable to a wide variety of patients. In addition, users with previous experience are likely to play with more of an entertainment attitude than learning [8].

\section{References}

1. Parker S (2011) Advancing the Field of Health Games. Health Games Research.

2. Peng W, Liu M (2009) An Overview of Using Electronic Games for Health Purposes. In An Overview of Using Electronic Games for Health Purposes.
3. Witteman DT (2011) Assessing the Acceptability and Usability of an Interactive Serious Game in Aiding Treatment Decisions for Patients with Localized Prostate Cancer. J Med Internet Res 13: e4.

4. Wattanasoontorn V, Imma Boada R, Sbert M (2013) Serious games for health. Entertainment Computing.

5. Hughest T, Flatt J, Fu B, Butters M, Chang CC, et al. (2014) Interactive video gaming compared with health education in older adults with mild cognitive impairment: a feasibility study. Inter J Geriatr Psychiatry 29: 890-898.

6. Gibson V, Douglas M (2013) The experience of developing an interactive educational tool based on board games. Nurse Educ Today 2013: 1612-1616.

7. http://edutechwiki.unige.ch/en/Serious_game\#Limitations

8. Lieberman DA (2006) What can we learn from playing interactive games? Consequences 2006: 379-397). 